\title{
MORFOLOGIA POLÍNICA DE CHRYSOPHYLLUM L. (SAPOTACEAE)
}

\author{
Maria Antonia Correia Sento Sé de Souza ${ }^{1}$; Francisco de Assis Ribeiro dos Santos ${ }^{2}$ \\ e Paulino Pereira Oliveira ${ }^{3}$ \\ 1. Bolsista PIBIC/CNPq, Graduando em Ciências Biológicas, Universidade Estadual de Feira de Santana, e-mail: \\ mari_uefs@yahoo.com.br \\ 2. Orientador, Departamento de Ciências Biológicas, Universidade Estadual de Feira de Santana, e-mail: \\ fasantos@uefs.br@uefs.br \\ 3. Co- orientador, Departamento de Ciências Biológicas, Universidade Estadual de Feira de Santana, e-mail: \\ paulino.pereira@gmail.com
}

PALAVRAS-CHAVE: sapotaceae, chrysophyllum, palinologia.

\section{INTRODUÇÃO}

No Brasil, a família Sapotaceae está representada por 12 gêneros e 232 espécies, estimando-se para a Bahia a ocorrência em torno de 77 espécies distribuídas em 11 gêneros (Carneiro et al. 2016). O gênero Chrysophyllum L. é o segundo maior gênero de Sapotaceae em número de espécies, abrangendo em torno de 31 espécies na região neotropical (Pennington, 1990). Para a Bahia, são citadas até o momento 12 espécies (Carneiro et al. 2016). A maioria das espécies de Chrysophyllum L. ocorre na América do Sul, principalmente em regiões de floresta úmida baixa. No Brasil, os centros de dispersão do gênero são Amazônia, principalmente e região costeira, frequentemente ocorrendo na Bahia, Espírito Santo, Rio de Janeiro e São Paulo (Pennington, 1991). Em se tratando de estudos palinológicos, o trabalho mais completo sobre Sapotaceae, foi realizado por Harley (1991) para espécies neotropicais, entretanto com carência de amostragem, sendo insuficiente para auxiliar na caracterização dos táxons. Com o avanço da tecnologia e o surgimento de outras ferramentas para auxiliar na taxonomia dos grupos, há a necessidade de levantar o maior número de informações sobre o táxon em foco de modo a contribuir significativamente para o conhecimento da flora como um todo, propiciando a sua identificação, utilização e conservação. Estudos palinológicos para o gênero são inexistentes, justificando a sua provável contribuição principalmente para a delimitação das suas espécies. O presente estudo foi desenvolvido com o objetivo de caracterizar a morfologia polínica das espécies do gênero Chysophyllum L. ocorrentes no estado da Bahia, visando identificar caracteres informativos que podem ajudar a aumentar o conhecimento sobre o gênero e subgênero deste grupo de plantas, além de contribuir para melhor delimitação taxonômica das mesmas.

\section{MATERIAL E MÉTODOS}

O levantamento das espécies foi feito através de consulta a herbário e bibliografia específica. A coleta de material botânico (botões florais) foi realizada em duplicatas depositadas no HUEFS (Herbário da Universidade Estadual de Feira de Santana). Os botões florais foram processados utilizando a técnica da acetólise de Erdtman (1960). As lâminas semi-permanentes com os grãos de pólen foram analisadas quantitativa e qualitativamente em microscopia óptica. Os dados obtidos foram utilizados para a descrição palinológicas das espécies, baseada em Barth e Melhem (1988), e a terminologia adotada seguiu a nomenclatura de Punt et al (2007). Dessa forma, os táxons foram ilustrados, destacando os caracteres palinológicos mais significativos taxonomicamente. 


\section{RESULTADOS E/OU DISCUSSÃO}

No presente trabalho foram analisadas oito espécies de Chrysophyllum L., das 12 citadas até o momento para a Bahia, sendo estas: Chrysophyllum flexuosum Mart.; Chrysophyllum inornatum Mart.; Chrysophyllum januariense Eichler.; Chrysophyllum marginatum Hook. \& Arn; Chrysophyllum ramiflora Mart.; Chrysophyllum rufum Mart.; Chrysophyllum splendens Spreng. e Chrysophyllum viride Mart. \& Eichler.

A seguir são apresentadas as descrições da morfologia polínica das espécies estudadas.

\section{Chrysophyllum flexuosum Mart.}

Grãos de pólen mônades, médios, isopolares, subprolatos, âmbito subtriangular; 3-(4)colporados, colpo curto; endoaberturas lalongadas e costada. Exina psilada, sexina e nexina com espessura aproximadamente igual na região polar. Chrysophyllum inornatum Mart.

Grãos de pólen mônades, médios, prolatos, isopolares, âmbito subtriangular; 3colporados; com ectoabertura do tipo colpo longo e estreito, de fácil visualização; endoabertura lalongada, costada e protuberante. Exina microrreticulada a levemente estriada, sexina mais espessa que a nexina na região polar.

Chrysophyllum januariense Eichler.

Grãos de pólen mônades, médio, prolatos, achatados nos polos, adquirindo uma forma mais ou menos retangular; amb subcircular ou subquadrangular; 3-(4)-colporados, colpo curto, endoaberturas lalongadas, costada e protuberante; Exina psilada, sexina e nexina com espessura aproximadamente igual na região polar.

Chrysophyllum marginatum (Hook. \& Arn.)

Grãos de pólen mônades, médios, prolatos, achatado nos polos, adquirindo uma forma mais ou menos retangular; 4-colporados; colpo longo, estreito; endoabertura lalongada, costada e protuberante; Exina microrreticulada; sexina mais espessa que a nexina na região polar, e com espessura aproximadamente igual na região equatorial.

Chrysophyllum ramiflora Mart.

Grãos de pólen mônades, médios, prolatos, isopolares, âmbito subtriangular; 3colporados; colpo longo; endoabertura lalongada, costada e protuberante. Exina microrreticulada, nexina de espessura semelhante à sexina na região polar.

Chrysophyllum rufum Mart.

Grãos de pólen mônades, médios, prolatos, âmbito subtriangular; 3-colporados; colpo longo, estreito; endoabertura lalongada, costada. Exina psilada, nexina e sexina de espessura semelhantes na região polar.

Chrysophyllum splendens Spreng.

Grãos de pólen mônades, médios, prolatos, âmbito subtriangular; 3-colporados; colpo curto, estreito; endoabertura lalongada, costada e protuberante. Exina: psilada e muito fina.

Chrysophyllum viride Mart. \& Eichler.

Grãos de pólen mônades, médios, prolatos; 4-colporados; ectoabertura do tipo colpo longo; endoabertura lalongada, costada e protuberante. Exina microrreticulada, nexina e sexina de espessura semelhantes na região polar.

Do ponto de vista palinológico, as espécies de Chrysophyllum L., aqui estudadas possuem grãos de pólen médios, com âmbito variando de subtriangular, subcircular a subquadrangular e exina variando de psilada a microreticulada; em geral, são prolatos (exceto em C. flexuosum onde são subprolatos), 3- (4)-colporados, com a endoabertura lalongada e costada. Todas as espécies aqui estudas tiveram a nexina, mais espessa do que a sexina; ao nível da endoabertura, a sexina separa-se consideravelmente da nexina que forma a costa ao redor da endoabertura. 


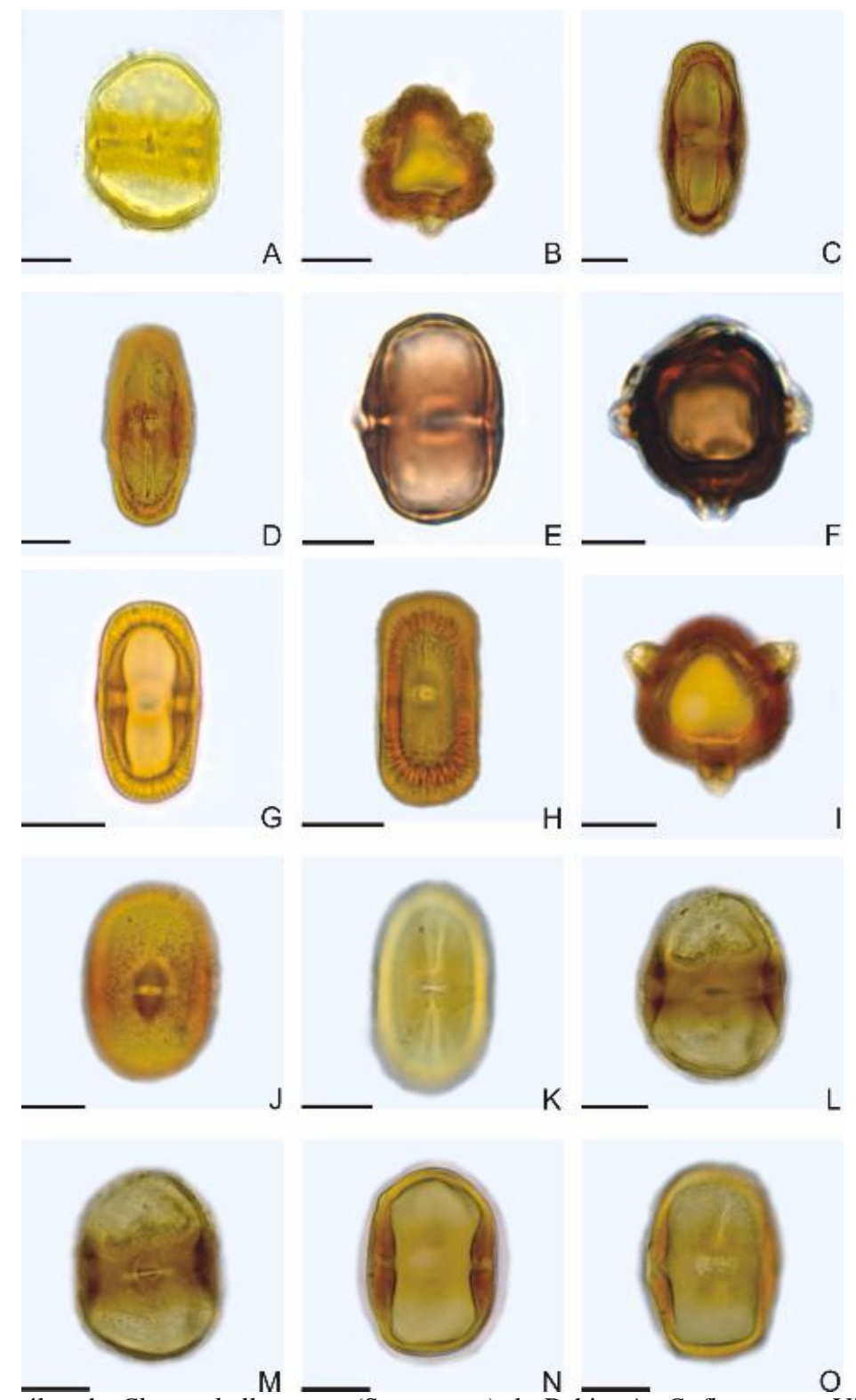

Figura 1. Grãos de pólen de Chrysophyllum spp. (Sapotaceae) da Bahia. A: C. flexuosum -Vista Equatorial, corte ótico; B-D: C. inornatum, B- Vista polar; C- Vista equatorial, corte ótico; D- Vista equatorial, superfície; E- $C$. januariense (Vista equatorial, corte ótico); F- C. januariense (Vista polar); G- C. marginatum (Vista equatorial, corte ótico); H- C. marginatum (Vista equatorial, superfície); I- C. ramiflora (Vista polar); J- C. ramiflora (Vista equatorial, superfície); K- C. rufum (Vista equatorial, superfície); L- C. splendens (Vista equatorial, corte ótico); MC. splendens (Vista equatorial, superfície); N- C. viride (Vista equatorial, corte ótico); O- C. viride (Vista equatorial, superfície). Barra $=10$

Tabela 2. Medidas (em $\mu \mathrm{m}$ ) dos grãos de pólen das espécies de Chrysophyllum spp. (Sapotaceae), para a Bahia.

\begin{tabular}{lcccc}
\hline \multicolumn{1}{c}{ Espécie } & \multicolumn{2}{c}{$\begin{array}{c}\text { Diâmetro polar }(\mu \mathrm{m}) \\
\text { DP }\end{array}$} & \multicolumn{2}{c}{ Diâmetro equatorial $(\mu \mathrm{m})$} \\
& $\begin{array}{l}\text { Faixa de } \\
\text { variação }\end{array}$ & $x$ & $\begin{array}{c}\text { Faixa de } \\
\text { variação }\end{array}$ \\
\hline C. flexuosum Mart. & $37,5-42,5$ & 39,2 & $27,5-32,5$ & 30,4 \\
C. inornatum Mart. & $27,5-32,5$ & 30,4 & $15,0-17,5$ & 16,3 \\
C. januariense Eichler. & $27,5-32,5$ & 29,9 & $17,5-25,5$ & 20,0 \\
C. marginatum Hook. \& Arn. & $25,0-30,0$ & 27,8 & $12,5-17,5$ & 15,3 \\
C. ramiflora Mart. & $27,5-35,0$ & 31,5 & $17,5-25,0$ & 20,8 \\
C. rufum Mart. & $22,5-30,0$ & 26,1 & $12,5-20,0$ & 16,0 \\
C. splendens Spreng. & $25,0-30,0$ & 27,2 & $17,5-22,5$ & 20,2 \\
C. viride Mart. \& Eichler. & $27,5-32,5$ & 28,6 & $15,0-22,5$ & 19,6 \\
\hline
\end{tabular}


Tabela 1. Caracteres morfométricos dos grãos de pólen das espécies de Chrysophyllum L. (Sapotaceae) ocorrentes na Bahia, Brasil.

\begin{tabular}{|c|c|c|c|}
\hline Espécie/Herbário & $\begin{array}{c}\text { Diâmetro polar } \\
(\mu \mathrm{m}) \\
\mathrm{DP} \\
\end{array}$ & $\begin{array}{c}\text { Diâmetro equatorial } \\
(\mu \mathrm{m}) \\
\mathrm{DE} \\
\end{array}$ & $\begin{array}{l}\text { Espessura da exina } \\
\qquad(\mu \mathrm{m})\end{array}$ \\
\hline \multicolumn{4}{|l|}{ Chrysophyllum flexuosum Mart. } \\
\hline $\begin{array}{l}\text { Matos, E.N. et al. } 188921 \text { (HUEFS ) } \\
\text { Chrysophyllum inornatum Mart. }\end{array}$ & 39,2 & 30,4 & 2,31 \\
\hline $\begin{array}{l}\text { Leite, K.R.B, R.P. et al. } 45677 \text { (HUEFS) } \\
\text { Chrysophyllum januariense Eichler. }\end{array}$ & 30,4 & 16,3 & 1,63 \\
\hline $\begin{array}{l}\text { Popovkin, A.V. } 198714 \text { (HUEFS) } \\
\text { Popovkin, A.V. } 198729 \text { (HUEFS ) }\end{array}$ & $\begin{array}{c}29,9 \\
7,1\end{array}$ & $\begin{array}{l}20,0 \\
22,9\end{array}$ & $\begin{array}{l}1,05 \\
1,05\end{array}$ \\
\hline $\begin{array}{l}\text { Chrysophyllum marginatum (Hook. \& } \\
\text { Arn.) }\end{array}$ & & & \\
\hline $\begin{array}{l}\text { Ferreira W.M. K. et al. } 8926 \text { (HUEFS) } \\
\text { Ganev, W. } 16576 \text { (HUEFS) }\end{array}$ & $\begin{array}{l}27,8 \\
24,9\end{array}$ & $\begin{array}{l}15,3 \\
15,0\end{array}$ & $\begin{array}{l}2,0 \\
2,0\end{array}$ \\
\hline Chrysophyllum ramiflora Mart. & & & \\
\hline $\begin{array}{l}\text { Bondar, G . } 68224 \text { (HUEFS) } \\
\text { Chrysophyllum rufum Mart. }\end{array}$ & 31,5 & 20,8 & 1,05 \\
\hline $\begin{array}{l}\text { Jardim, J.G. } 62859 \text { (HUEFS ) } \\
\text { Chrysophyllum splendens } \text { Spreng. }\end{array}$ & 26,1 & 16,0 & 0,53 \\
\hline $\begin{array}{l}\text { Cardoso, D.149417 (HUEFS ) } \\
\text { Chrysophyllum viride Mart. \& Eichler. }\end{array}$ & 27,2 & 20,2 & 0,53 \\
\hline Ferretti, A.R. et al. 117151 (HUEFS) & 28,6 & 19,6 & 1,05 \\
\hline
\end{tabular}

\section{CONSIDERAÇÕES FINAIS}

Com base na análise dos resultados apresentados e da bibliografia levantada, conclui-se que os grãos de pólen de Chrysophyllum spp. (Sapotaceae) para o estado da Bahia, tratase de um gênero estenopolínico, em que a morfologia polínica é invariável dentro do grupo, necessitando assim de uma caracterização mais detalhada destes grãos de pólen através de análises em microscopia eletrônica de varredura, por exemplo, o que poderá ser mais um subsídio para auxiliar na taxonomia do grupo. Deste modo, por ser um dos primeiros estudos na Bahia sobre a morfologia polínica do gênero, o presente estudo poderá subsidiar futuros trabalhos tanto local como regional, nas diversas áreas da Palinologia.

\section{REFERÊNCIAS}

BARTH, Ortrud Monika e Therezinha Sant'anna Melhem. Glossário Ilustrado de Palinologia. Campinas. 1988.

CARNEIRO, C.E.; Alves-Araujo, A.; Almeida Jr., E.B. Sapotaceae. In: Lista de Espécies da Flora do Brasil. Jardim Botânico do Rio de Janeiro. 2016.

ERDTMAN, G. The acetolysis method. A revised description. Svensk Botanisk Tidskrift. 1960

HALEY, Madeline M. The Pollen Morphology of the Sapotaceae. Vol. 46, No. 3. 1991.

PENNINGTON, T.D. Sapotaceae. In: Flora Neotropica, New York, v. 52, 770p. 1990. PENNINGTON, T.D.. The genera of Sapotaceae. London, Royal Botanic Gardens Kew, 307p. 1991

PUNT, W.: Hoen, P.P.: Blackmore, S.; Nilsson, S. e Le Thomas, A. Glossary of pollen and spore terminology. Review of Paleobotany and Palynology. 2007 